\title{
Percepção de pacientes sobre a comunicação de médicos clínicos e cirurgiões em hospital universitário
}

\section{Patient's perception of the communication of clinical doctors and surgeons in a university hospital}

\author{
Milena Regina Dos Santos Perez ${ }^{1}$ (1) milenah72@hotmail.com \\ Mirele Cristine Santos de Oliveira² (1) mirele.santos@live.com \\ Danielle Bispo Vieira Ortiz ${ }^{1}$ (D) danielle.bvieira@gmail.com \\ Stela Souza Peña' (D) stela.pena@hotmail.com \\ Jose Roberto Pretel Pereira Job' (1) preteljob2@yahoo.com.br \\ Reinaldo José Gianini' (D) rgianini@pucsp.br
}

\begin{abstract}
RESUMO
Introdução: A maneira de transmitir informações é essencial na relação médico-paciente, pois a boa comunicação reduz queixas por práticas inadequadas e preocupações dos pacientes, e melhora a adesão aos tratamentos e a recuperação da saúde. Porém, não são raras as insatisfações dos pacientes sobre esse assunto.

Objetivo: O objetivo deste trabalho foi avaliar a percepção dos pacientes internados em um complexo hospitalar sobre a comunicação de médicos clínicos e cirurgiões durante o período de internação.

Método: Trata-se de estudo transversal, descritivo e analítico, do tipo inquérito, com aplicação de um questionário com perguntas sobre a comunicação geral do médico. O instrumento foi construído pelos pesquisadores e respondido por 120 pacientes adultos. A amostra foi definida por conveniência, estratificada por clínica médica e cirúrgica. Realizaram-se análises de frequência e estatística dos resultados encontrados.

Resultados: Dos 120 pacientes, 53,33\% ( $n=64)$ foram internados na clínica cirúrgica e $46,67 \%(n=56)$ na clínica médica. Desse total, 57,5\% $(n=69)$ tinham escolaridade que variava de ensino médio a superior. Os pacientes relataram respostas mais negativas do que positivas em questões referentes a: informações sobre os efeitos colaterais dos medicamentos (66\%), orientações de procedimentos pós-cirúrgicos (68,75\%) e informações sobre promoção e prevenção da saúde no ambiente hospitalar (63,33\%). A clínica cirúrgica teve proporções de respostas positivas significativamente menores para: "O médico disse o nome dele" ( $\mathrm{p}<0,01$; OR bruta 0,33; IC95\% 0,15-0,76); "O paciente foi informado sobre como será seu tratamento" ( $p=0,02$; OR bruta 0,38; IC95\% 0,17-0,87); e "O paciente foi informado sobre a necessidade de realizar exames" ( $p=0,02$; OR bruta 0,40; IC95\% 0,18-0,90), que se mantiveram significativas após o ajuste por determinados fatores intervenientes. Não se observaram diferenças significativas para as demais questões. Na análise da questão "Que nota você daria para a comunicação geral do médico? ", verificou-se valor significativamente maior ( $p=0,007$ ) para a clínica médica (média 4,46 $\pm 0,76$ ) quando comparada à clínica cirúrgica (média $4 \pm 1,19$ ).

Conclusão: A comunicação médico-paciente apresentou déficits significativos. Por isso, é necessário que as escolas médicas ofereçam para os discentes o desenvolvimento dessa competência. Além disso, para uma generalização adequada dos resultados encontrados, novos estudos precisam ser realizados em níveis diferentes do cuidado médico.
\end{abstract}

Palavras-chave: Especialidade Médica; Comunicação em Saúde; Relações Médico-Paciente.

\begin{abstract}
Introduction: The way information is transmitted is of crucial importance in the doctor-patient relationship, as good communication reduces complaints about inadequate practices and patient concerns and improves treatment adherence and health recovery. However, patient dissatisfaction on this subject is not unusual.

Objectives: The objective of this work was to evaluate the perception of patients admitted to a Hospital Complex about the communication of clinical doctors and surgeons during the hospitalization period.

Method: Cross-sectional, descriptive, analytical inquiry study, with the application of a questionnaire with questions about physicians' general communication. The instrument was built by the researchers and was answered by 120 adult patients. The sample was defined by convenience and stratified by medical and surgical clinic. Frequency and statistical analyses were performed on the obtained results.

Results: Of 120 patients, $53.33 \%(n=64)$ were admitted to the Surgical Clinic and $46.67 \%(n=56)$ to the Medical Clinic. Of this total, $57.5 \%(n=69)$ had high school to college/university education. Patients reported more negative than positive responses to the following questions: information about the side effects of medications (66\%), advice on post-surgical procedures $(68.75 \%)$ and information on health promotion and prevention in the hospital environment (63.33\%). The surgical clinic had significantly lower proportions of positive responses for: The doctor said their name ( $p<0.01 ; \mathrm{crude}$ OR: 0.33; 95\% Cl 0.15-0.76); The patient was informed about how their treatment would be conducted ( $p=0.02 ; \mathrm{crude}$ OR: 0.38; 95\% Cl 0.17-0.87); and the patient was informed about the need to undergo tests ( $p=0.02$; crude OR 0.40; 95\% Cl 0.18-0.90), which remained significant after adjustment for certain confounding factors. There were no significant differences regarding the other questions. When analyzing the question: "What grade would you give to the doctor's general communication?" a significantly higher value $(p=0.007)$ was given to the Medical Clinic (average $4.46 \pm 0.76)$ when compared to the Surgical Clinic (average 4 \pm 1.19 ).
\end{abstract}

Conclusion: The doctor-patient communication showed significant deficits. Therefore, it is necessary for medical schools to offer students the development of this competence. Additionally, for an adequate generalization of the obtained results, new studies need to be carried out at different levels of medical care. Keywords: Medical Specialties; Health Communication; Doctor-Patient Relationships.

${ }^{1}$ Pontifícia Universidade Católica de São Paulo, Sorocaba, São Paulo, Brasil.

2 Universidade de Marília, Marília, São Paulo, Brasil.

Editora-chefe: Rosiane Viana Zuza Diniz ～～Editor Associado: Kristopherson Lustosa Augusto

Recebido em 10/24/20; Aceito em 03/01/21. | Avaliado pelo processo de double blind review. 


\section{INTRODUÇÃO}

As Diretrizes Curriculares para os Cursos de Graduação em Saúde no Brasil visam construir um perfil acadêmico e profissional de médicos com habilidades que se encaixem na abordagem do Sistema Único de Saúde (SUS). Então, o profissional deve ser capaz de agir com qualidade, eficiência e resolutividade ${ }^{1}$. Nesse contexto, a linguagem do médico deve ser acessível aos pacientes, colegas de trabalho e familiares, por meio de comunicação adequada para cada grupo. Essa maneira de transmitir informações é essencial na relação médico-paciente, pois a boa comunicação reduz as queixas por práticas inadequadas e preocupações dos pacientes, e melhora a adesão aos tratamentos e a recuperação da saúde tanto física quanto mental².

Também no cenário mundial, a capacidade de se comunicar com o paciente é indispensável, fazendo parte de programas obrigatórios para residentes nos Estados Unidos, como no Exame de Licenciamento Médico ${ }^{3}$.

No entanto, a insatisfação dos pacientes em relação à comunicação com profissionais médicos não é incomum. Os pacientes queixam-se do uso de jargões excessivamente técnicos e pouco compreensíveis, da incapacidade de receber informações suficientes para tirar conclusões do estado clínico, da frieza demonstrada pelo profissional médico diante da situação do paciente, entre outros. Esses motivos podem levar a reclamações nos conselhos médicos e a ações judiciais ${ }^{4}$.

Nesse sentido, um estudo realizado por Loge et al. ${ }^{5}$ observou que a maneira como a informação era transmitida limitava o entendimento sobre as questões de saúde em 497 pacientes com câncer de mama. De acordo com Scott et al. ${ }^{6}$, muitas pessoas, ao receberem um diagnóstico de sua patologia, têm dificuldade em lembrar as informações fornecidas durante a consulta. Uma metanálise mostrou que a comunicação médica tem um impacto positivo para maior adesão dos pacientes ao tratamento ${ }^{7}$.

Portanto, identificar as características da comunicação, principalmente por pacientes hospitalizados, é fundamental para fortalecer a importância do tema aos membros desse ambiente profissional.

O objetivo deste estudo é avaliar a percepção dos pacientes sobre a comunicação de médicos clínicos e cirurgiões durante o período de internação, especificamente: 1. Apresentação do profissional: conhecimento do nome e da especialidade; 2. Esclarecimento sobre diagnósticos, exames, terapêutica, evolução e conduta; 3. Aplicação do Termo de Consentimento Informado; 4. Orientações acerca de ações terapêuticas e diagnósticas durante a internação; 5. Pacientes cirúrgicos: informações relacionadas ao pré-operatório (como anestesia e medicamentos) e pós-operatório (como efeitos colaterais e cuidados pessoais); 6. Orientação sobre medidas preventivas e promotoras de saúde no ambiente hospitalar; 7. Qualidade geral da comunicação médica.

\section{MÉTODOS}

Trata-se de estudo transversal, descritivo e analítico, do tipo inquérito, com a aplicação de um questionário, construído pelos pesquisadores, com o objetivo de analisar as percepções de pacientes internados no Conjunto Hospitalar de Sorocaba (CHS) sobre a comunicação médica durante o período de hospitalização̊ .

\section{População de referência}

Administrado pelo Serviço Social da Construção Civil do Estado de São Paulo (Seconci-SP), o complexo do CHS compreende os hospitais Prof. Dr. Lineu Matos Silveira, Leonor Mendes de Barros e o Ambulatório Nelson da Costa Chaves. O complexo soma mais de 2.200 colaboradores e é responsável pelo atendimento do nível terciário de 48 municípios do sudoeste paulista, com uma população de mais de três milhões de habitantes. A unidade possui 450 leitos: 350 fixos e 100 móveis. A taxa de ocupação dos leitos na internação é de aproximadamente 93,6\% na clínica médica e $83,1 \%$ na clínica cirúrgica 9 .

\section{Amostra}

A amostra foi definida por conveniência, estratificada por clínica médica e cirúrgica, com um total esperado de 120 pacientes a serem entrevistados. Não houve cálculo amostral.

Os pacientes eram convidados a participar do estudo quando o entrevistador entrava em quartos aleatórios e explicava verbalmente o projeto. Evitou-se a abordagem de pacientes visivelmente graves, com medicações sedativas e alteração do nível de consciência. Essa verificação era feita rapidamente, com perguntas sobre a orientação auto e alopsíquica do paciente.

Foram entrevistados pacientes internados por, no mínimo, dois dias na clínica médica e na clínica cirúrgica, independentemente da especialidade, e que assinaram o Termo de Consentimento Livre e Esclarecido (TCLE). Os pacientes precisavam ter 18 anos ou mais e estar em condições mentais e emocionais para participar.

Excluíram-se os pacientes menores de 18 anos, aqueles que não estavam em condições mentais para responder, que não forneceram informações verdadeiras sobre sua condição geral e seus dados sociodemográficos, os que estavam havia menos de dois dias internados e os que não estavam interessados em participar do estudo. Não se fez análise dos prontuários dos pacientes. 


\section{Questionário}

O questionário foi construído para o presente estudo com perguntas intervalares, segundo a escala Likert, e dicotômicas (opções sim / não), que totalizaram 25 questões. Para sua construção, foram selecionados temas relacionados à comunicação médica, tendo como referência as Diretrizes Curriculares Nacionais para os Cursos de Graduação em Medicina do Ministério da Educação do Brasil ${ }^{10}$. Na clínica cirúrgica, houve um tópico relacionado ao procedimento cirúrgico. O questionário utilizado na pesquisa passou por um pré-teste com 15 pacientes, para serem feitas as correções necessárias em sua aplicação. Não ocorreu procedimento de validação.

As questões referentes à percepção dos pacientes sobre a comunicação médica foram, em sua maioria, do tipo sim ou não - e, por isso, não há modulação.

\section{Variáveis e categorias}

Os dados sociodemográficos coletados foram divididos em grupos: idade (mais de 45 anos e menos de 45 anos), sexo (feminino e masculino), etnia (branco/amarelo e pardo/ negro), estado civil (uniões formais/informais e solteiro/ separado/viúvo), escolaridade (fundamental, médio, superior) e procedência (Sorocaba e outros municípios). Na categoria informações sobre internação, registraram-se o motivo da internação do paciente e o tempo de internação.

Para a categoria de avaliação da comunicação médica, questionaram-se os seguintes aspectos: dados em relação ao sexo do médico; como este se apresentou ao paciente; se a especialidade do médico foi informada - se sim, qual era; como o médico se referiu ao paciente; se questionou sobre possíveis dúvidas - e, em caso afirmativo, se elas foram esclarecidas; se o diagnóstico foi esclarecido pelo médico; se se explicou a necessidade da internação e se o paciente foi informado sobre como seria o tratamento; se foi explicada a necessidade de realização de exames; se o paciente concordava com o procedimento e se assinou o TCLE; se foi informado sobre possíveis complicações do tratamento e recebeu orientações sobre promoção e prevenção de saúde no ambiente hospitalar. Nesse último tópico, questionou-se verbalmente se o médico informou o paciente sobre deambulação, decúbito inclinação no leito, com o intuito de evitar possíveis complicações advindas da imobilização prolongada, como úlceras por pressão ou trombose venosa profunda. Ao final, perguntou-se ao paciente que nota ele daria para a comunicação geral do médico, sendo as opções de resposta: ótima, boa, regular, ruim ou péssima.

Especificamente, os pacientes internados na enfermaria cirúrgica foram questionados se receberam informações sobre: tipos de anestesia, medicamentos na cirurgia, riscos e benefícios da realização da cirurgia, cuidados pessoais e de higiene no pós-cirúrgico e possíveis efeitos colaterais após a realização da cirurgia.

\section{Procedimento de coleta de dados}

Na aplicação do questionário, adotaram-se as seguintes etapas: o pesquisador responsável explicou a pesquisa e, enquanto esperava a confirmação de participação do entrevistado, leu o questionário, além de solicitar a assinatura doTCLE. Cada paciente indicou sua opinião sobre cada questão, além de informar sobre as questões sociodemográficas. Escolheu-se a abordagem das respostas a serem marcadas pelo pesquisador, após o paciente ser questionado verbalmente, para evitar dados ausentes. A coleta de dados ocorreu de março a novembro de 2018 , no leito do paciente.

\section{Procedimento da análise dos dados}

Tabularam-se os dados coletados em uma planilha no Excel, e realizaram-se testes de hipóteses qui-quadrado, do programa Stata 16.0, com avaliação da significância das hipóteses levantadas ${ }^{11}$.

Realizou-se análise da distribuição de frequências absoluta e relativa das variáveis pesquisadas, além de testes de associação (qui2) entre respostas questionário/ sociodemográfico com especialidade.

Fizeram-se as regressões logísticas uni e multivariada com cálculo da odds ratio (OR) e respectivos intervalos de confiança (IC) de 95\%. Além disso, foi realizado ajuste para variáveis potenciais de confundimento.

Para a nota da comunicação geral do médico, realizou-se o teste $t$ de Student.

Foi realizada, indiretamente, uma análise de sensibilidade por meio do ajuste por variáveis associadas à percepção do paciente sobre a comunicação médica, que constam na Tabela 2.

\section{Ética}

O presente estudo foi aprovado pelo Comitê de Ética em Pesquisa da Pontifícia Universidade Católica de São Paulo (PUCSP), campus de Sorocaba - Certificado de Apresentação para Apreciação Ética (CAAE) n 83741918.0.0000.5373.

\section{RESULTADOS}

Dos 120 pacientes, $53,33 \%(n=64)$ foram internados na clínica cirúrgica e $46,67 \%(n=56)$ na clínica médica. Desse total, $57,5 \%(n=69)$ tinham ensino médio ou superior $e$ $47,5 \%(n=51)$ haviam cursado até o ensino fundamental. Os pacientes entrevistados eram procedentes de 29 municípios distintos, todos da regional de saúde. O período das entrevistas ocorreu de 9 de abril a 15 de dezembro de 2018, entre 8 e 18 horas. 
A distribuição da amostra, segundo os possíveis fatores intervenientes e a análise de suas associações com o tipo de clínica (médica ou cirúrgica), apresentou diferença significativa apenas para a variável gênero, com predominância de homens na clínica médica $(p=0,04)$. Não se observaram diferenças para as outras variáveis sociodemográficas, como também não se verificou diferença para o tempo de internação ou gênero do médico assistente (Tabela 1).

$\mathrm{Na}$ análise da associação entre as questões que avaliaram a percepção do paciente sobre a comunicação do médico e a clínica, verificaram-se diferenças significativas entre clínica cirúrgica e clínica médica. A clínica cirúrgica teve proporções de respostas positivas significativamente menores para: "O médico disse o nome dele" ( $p<0,01$; OR bruta 0,33; IC95\% 0,15-0,76); "O paciente foi informado sobre como será seu tratamento" ( $p=0,02$; OR bruta 0,38; IC95\% $0,17-0,87) ;$ e "O paciente foi informado sobre a necessidade de realizar exames" ( $p=0,02$; OR bruta 0,40; IC95\% 0,18$0,90)$, que se mantiveram significativas após o ajuste por determinados fatores intervenientes. Não se observaram diferenças significativas para as demais questões. A única variável que apresentou diferença de mais de $10 \%$ entre OR bruta e ajustada foi "O médico explicou sobre a necessidade da internação" (OR bruta = 1,00; OR ajustada por gênero = 1,30; ambas não significativas - $p>0,05$ ) (Tabela 2).

$\mathrm{Na}$ análise da questão "Que nota você daria para a comunicação geral do médico?", verificou-se valor significativamente maior ( $p=0,007$ ) para a clínica médica (média 4,46/desvio padrão 0,76 ) quando comparada à clínica cirúrgica (média 4/desvio padrão 1,19).

Quanto à análise das questões específicas aplicadas à clínica cirúrgica, observou-se que apenas a informação sobre efeitos colaterais atingiu a maioria dos pacientes, ficando os demais itens com proporção bem menor.

Tabela 1. Distribuição da amostra segundo possíveis fatores intervenientes e análise de suas associações com o tipo de clínica.

\begin{tabular}{|c|c|c|c|}
\hline Fator & $\begin{array}{c}\text { Clínica médica } \\
\text { N (\%) }\end{array}$ & $\begin{array}{c}\text { Clínica cirúrgica } \\
\mathrm{N}(\%)\end{array}$ & $\mathbf{P}$ \\
\hline Gênero & & & 0,04 \\
\hline Masculino & $33(58,9)$ & $26(40,6)$ & \\
\hline Feminino & $23(41,1)$ & $38(59,4)$ & \\
\hline Idade & & & 0,26 \\
\hline$<45$ anos & $24(42,8)$ & $34(53,1)$ & \\
\hline 45 ou mais & $32(57,2)$ & $30(46,9)$ & \\
\hline Etnia/raça & & & 0,61 \\
\hline Branco/amarelo & $28(50,0)$ & $29(45,3)$ & \\
\hline Negro/pardo & $28(50,0)$ & $35(54,7)$ & \\
\hline Estado civil & & & 0,79 \\
\hline Uniões formais ou informais & $33(58,9)$ & $37(57,8)$ & \\
\hline Solteiro, separado ou viúvo & $23(41,1)$ & $27(42,2)$ & \\
\hline Escolaridade & & & 0,50 \\
\hline Médio ou superior & $34(60,7)$ & $35(54,7)$ & \\
\hline Fundamental & $22(39,3)$ & $29(45,3)$ & \\
\hline Procedência & & & 0,89 \\
\hline Sorocaba & $16(28,6)$ & $19(29,7)$ & \\
\hline Outros municípios da região & $40(71,4)$ & $45(70,3)$ & \\
\hline Tempo de internação & & & 0,70 \\
\hline$<8$ dias & $30(53,6)$ & $32(50,0)$ & \\
\hline 8 dias ou mais & $26(46,4)$ & $32(50,0)$ & \\
\hline Gênero do médico & & & 0,74 \\
\hline Masculino & $20(35,7)$ & $24(37,5)$ & \\
\hline Feminino & $36(64,3)$ & $40(62,5)$ & \\
\hline Total & $56(100,0)$ & $64(100,0)$ & \\
\hline
\end{tabular}

Fonte: Elaborada pelos autores. 
Tabela 2. Análise da associação entre as questões que avaliaram a percepção do paciente sobre a comunicação do médico e a clínica.

\begin{tabular}{|c|c|c|c|c|c|c|}
\hline Questão & $\begin{array}{l}\text { Clínica } \\
\text { Sim/não }\end{array}$ & $\begin{array}{l}\text { Cirúrgica } \\
\text { Sim/não }\end{array}$ & $\mathbf{P}$ & $\begin{array}{l}\text { OR e IC95\% } \\
\text { brutos } \\
\text { Cirúrgica/clínica }\end{array}$ & $\begin{array}{c}\text { OR e IC95\% } \\
\text { ajustados } \\
\text { Cirúrgica/clínica }\end{array}$ & $\begin{array}{c}\text { Variáveis } \\
\text { no modelo* }\end{array}$ \\
\hline O médico disse o nome dele. & $45 / 11$ & $37 / 27$ & $<0,01$ & $\begin{array}{c}0,33 \\
0,15-0,76\end{array}$ & $\begin{array}{c}0,36 \\
0,15-0,87\end{array}$ & $1,2,4$ \\
\hline A especialidade foi informada. & $32 / 24$ & $25 / 29$ & 0,16 & $\begin{array}{c}0,51 \\
0,24-1,06\end{array}$ & $\begin{array}{c}0,54 \\
0,26-1,15\end{array}$ & 1 \\
\hline $\begin{array}{l}\text { O médico perguntou se o } \\
\text { paciente tinha alguma dúvida. }\end{array}$ & $33 / 23$ & $34 / 30$ & 0,52 & $\begin{array}{c}0,79 \\
0,38-1,63\end{array}$ & $\begin{array}{c}0,87 \\
0,41-1,82\end{array}$ & 1 \\
\hline O médico informou o diagnóstico. & $45 / 11$ & $54 / 10$ & 0,56 & $\begin{array}{c}1,32 \\
0,51-3,39\end{array}$ & $\begin{array}{c}1,35 \\
0,52-3,53\end{array}$ & 1 \\
\hline $\begin{array}{l}\text { O médico explicou sobre a } \\
\text { necessidade da internação }\end{array}$ & $49 / 7$ & $56 / 8$ & 1,0 & $\begin{array}{c}1,00 \\
0,34-2,96\end{array}$ & $\begin{array}{c}1,30 \\
0,42-4,04\end{array}$ & 1 \\
\hline $\begin{array}{l}\text { O paciente foi informado sobre } \\
\text { como será o tratamento. }\end{array}$ & $45 / 11$ & $39 / 25$ & 0,02 & $\begin{array}{c}0,38 \\
0,17-0,87\end{array}$ & $\begin{array}{c}0,40 \\
0,17-0,93\end{array}$ & 1,4 \\
\hline $\begin{array}{l}\text { O paciente foi informado sobre a } \\
\text { necessidade de realizar exames. }\end{array}$ & $44 / 12$ & $38 / 26$ & 0,02 & $\begin{array}{c}0,40 \\
0,18-0,90\end{array}$ & $\begin{array}{c}0,39 \\
0,17-0,91\end{array}$ & 1,3 \\
\hline $\begin{array}{l}\text { O médico perguntou se concordava } \\
\text { com o tratamento/cirurgia. }\end{array}$ & $32 / 24$ & $30 / 34$ & 0,26 & $\begin{array}{c}0,66 \\
0,32-1,36\end{array}$ & $\begin{array}{c}0,63 \\
0,30-1,31\end{array}$ & 1 \\
\hline $\begin{array}{l}\text { O paciente foi informado sobre } \\
\text { possíveis efeitos colaterais. }\end{array}$ & $30 / 26$ & $24 / 40$ & 0,08 & $\begin{array}{c}0,52 \\
0,25-1,08\end{array}$ & $\begin{array}{c}0,54 \\
0,26-1,13\end{array}$ & 1 \\
\hline $\begin{array}{l}\text { O médico informou sobre a } \\
\text { evolução do caso. }\end{array}$ & $43 / 13$ & $41 / 23$ & 0,13 & $\begin{array}{c}0,54 \\
0,24-1,20\end{array}$ & $\begin{array}{c}0,52 \\
0,23-1,18\end{array}$ & 1 \\
\hline $\begin{array}{l}\text { O médico orientou sobre } \\
\text { promoção e prevenção de saúde } \\
\text { no ambiente hospitalar. }\end{array}$ & $22 / 34$ & $22 / 42$ & 0,58 & $\begin{array}{c}0,81 \\
0,38-1,70\end{array}$ & $\begin{array}{c}0,78 \\
0,36-1,69\end{array}$ & 1,5 \\
\hline
\end{tabular}

*1 = local; 2 = idade; 3 = raça; 4 = escolaridade; 5 = tempo de internação.

Fonte: Elaborada pelos autores.

Tabela 3. Descrição das respostas às questões específicas aplicadas aos pacientes da clínica cirúrgica $(\mathrm{N}=64)$.

\begin{tabular}{lc}
\hline \multicolumn{1}{c}{ Questão } & SIM/NÃO \\
\hline O Sr./a Sra. foi informado(a) sobre tipo de anestesia e medicamentos? & $25 / 39$ \\
O Sr./a Sra. foi informado(a) sobre riscos e benefícios de realizar a cirurgia? & $24 / 40$ \\
O Sr./a Sra. foi informado(a) sobre cuidados especiais com a ferida cirúrgica e questões de higiene no & $27 / 37$ \\
pós-cirúrgico? & $44 / 20$ \\
\hline
\end{tabular}

Fonte: Elaborada pelos autores.

\section{DISCUSSÃO}

Como os pacientes não eram informados sobre inclinação do leito, mudança de decúbito, deambulação e orientação sobre hábitos de vida, as respostas foram mais negativas do que positivas nas seguintes perguntas: informações sobre os efeitos colaterais dos medicamentos (66\%), orientações de procedimentos pós-cirúrgicos aos pacientes submetidos a cirurgias $(68,75 \%)$ e informações sobre promoção e prevenção da saúde no ambiente hospitalar (63,33\%). Percebe-se defasagem na promoção de saúde, que se afasta de projetos como o Health Promotion Hospital, construído com os seguintes objetivos: viabilizar a educação dos pacientes para reduzir riscos e problemas de saúde, promover a prevenção de doenças e abordar o estilo de vida do paciente no ambiente hospitalar ${ }^{12}$.

Segundo Razera et al. ${ }^{13}$, os pacientes precisam ser informados sobre os procedimentos cirúrgicos para que possam obter benefícios na saúde mental e física, como diminuição da ansiedade e angústias. Além disso, no estudo de Singer et al. ${ }^{14}$ que investigou a opinião dos pacientes sobre a qualidade do atendimento e suporte emocional recebidos durante o tratamento, verificou-se que as informações fornecidas antes dos procedimentos são um dos domínios mais citados como extremamente importantes.

Nossos dados mostram lacunas na comunicação médica 
em relação à doença dos pacientes e sobre o acompanhamento adequado que os médicos deveriam realizar, o que foi observado também por Schaller et al. ${ }^{15}$.

Uma metanálise sobre o impacto da comunicação médica no processo de assistência à saúde concluiu que os pacientes mais bem informados têm melhor adesão ao tratamento, compreendem melhor sua patologia e se envolvem mais na tomada de decisões, com um aumento de mais de $19 \%$ de adesão a propostas terapêuticas ${ }^{7}$.

\section{Diferenças entre médicos clínicos e cirurgiões}

No presente estudo, a comunicação de médicos clínicos e cirurgiões apresentou diferença significativa $(p<0,05)$ nos seguintes tópicos: o médico dizer o nome dele, o paciente ser informado sobre como será o tratamento ou sobre a necessidade de realização de exames.

O estudo de Feba et al. ${ }^{16}$ avaliou os contrastes de personalidade de médicos clínicos e cirurgiões e constatou que aqueles tendem a ser mais sentimentais. Os cirurgiões, por sua vez, demonstraram maior objetividade no contato com os pacientes. De acordo com Kang et al. ${ }^{17}$, os cirurgiões são mais rápidos e impulsivos, e, apesar de mostrarem interesse no contato interpessoal, isso ocorreu com menor frequência em realação aos médicos clínicos, considerados mais detalhistas, reflexivos e imaginativos. Bellodi ${ }^{18}$ demonstrou a percepção das características das diferentes especialidades e seu impacto na forma de atendimento ao paciente, ilustrando a praticidade e cautela nas relações interpessoais dos cirurgiões, com manipulação objetiva e concreta da realidade. Por sua vez, os médicos clínicos foram definidos como prudentes, vigilantes e consultores.

Essas definições são importantes na maneira como as informações são transmitidas ao paciente e à família, pois um profissional com comunicação mais atenta pode facilitar o processo de orientação sobre procedimentos e terapias, e garantir que o menor número de dúvidas permaneça após o contato com o paciente. Separando as especialidades clínicas e cirúrgicas, um estudo alemão constatou que 39\% dos clínicos trabalhavam mais de 60 horas por semana, enquanto $68 \%$ dos cirurgiões trabalhavam essa média, podendo demonstrar a maneira diferente de abordar o paciente como reflexo de uma intensa e estressante jornada de trabalho ${ }^{19}$.

Considerando que muitos dos profissionais médicos que atendem no CHS podem realizar atendimentos emergenciais, como de pacientes politraumatizados, é importante compreender as condições de trabalho e seu impacto na qualidade do atendimento prestado, já que a demanda aumentada em serviços de urgência e emergência pode gerar desorganização dentro da unidade, atendimento de baixa qualidade, gastos desnecessários e insatisfações por parte dos trabalhadores e dos pacientes ${ }^{20}$.

Apesar dos pontos negativos na comunicação médicopaciente visíveis nos resultados obtidos neste estudo (não esclarecimento sobre os efeitos de medicamentos $\mathrm{e}$ procedimentos, promoção da saúde e questões relacionadas aos procedimentos cirúrgicos), muitos entrevistados (81\%) consideraram a comunicação médica excelente ou boa. Quando perguntados se o médico questionou se o paciente tinha alguma dúvida, 55,8\% dos pacientes afirmaram ter recebido essa abordagem, com um nível de esclarecimento de dúvidas de $97 \%$. No entanto, houve diferença nas notas entre clínicos e cirurgiões ( $p=0,007)$, com a nota mais alta na comunicação oferecida aos profissionais da clínica médica.

\section{Apresentação do profissional médico}

Em relação à apresentação do profissional médico informando o nome e a especialidade, embora a maioria dos pacientes tenha relatado que foi dito o nome do médico, o teste de hipótese encontrou associação estatisticamente significativa entre a falta de informações sobre o nome e a especialidade do profissional de saúde e a área de atuação $(p<0,01)$.

A não apresentação do médico é um ponto negativo destacado nos estudos publicados por Roter ${ }^{21}$ que definiram a necessidade de o profissional se apresentar ao paciente, cumprimentá-lo e criar um ambiente confiável e construtivo no relacionamento, de modo a facilitar a troca de informações. No entanto, outros autores destacaram outros problemas relacionados à comunicação dos médicos. Por exemplo, Sheppard et al..$^{22}$ mostraram que muitos pacientes hospitalizados apresentaram problemas de comunicação com profissionais médicos, que demonstraram despreparo para discutir as opções de tratamento. Condição semelhante foi observada por Endo et al. $^{23}$, em que a maior barreira identificada no atendimento a pacientes com câncer de pulmão foi a falta de informações corretas ou de entendimento sobre as opções de tratamento oferecidas. Arora et al. ${ }^{24}$ e Singer et al. ${ }^{14}$ relataram a importância do apoio emocional ao paciente para manter a confiança no médico durante todo o tratamento, além de melhorar sua saúde mental.

Da mesma forma, Ruberton et al. ${ }^{25}$ e Schaller et al. ${ }^{15}$ demonstraram associação entre a qualidade da comunicação médica e a satisfação do paciente com os cuidados recebidos. Os médicos definidos como menos arrogantes obtiveram melhores notas, de acordo com a satisfação do paciente.

\section{Termo de Consentimento Livre e Esclarecido}

A aplicação do TCLE por parte dos profissionais médicos, embora verbal, não ocorreu em quase metade dos pacientes entrevistados, mas há uma tendência de não aplicação pelas diferentes especialidades médicas. 
Em estudo realizado no Hospital Universitário da Universidade Federal de Sergipe, não encontrou o TCLE nos prontuários de pacientes submetidos a intervenções invasivas. Entre as justificativas observadas, os médicos relataram não ter tempo para a aplicação devido ao volume excessivo de consultas, apesar de temerem que os pacientes se sentissem inseguros na realização do procedimento ${ }^{12}$.

A forma verbal é mais utilizada na maioria dos procedimentos, seguida do registro no prontuário. No entanto, a recomendação do Conselho Federal de Medicina é que exista uma elaboração escrita do termo. O TCLE deve ser um documento em que o paciente ou o representante legal seja informado sobre a doença, as alternativas de tratamento, os efeitos adversos esperados e o prognóstico ${ }^{26}$.

Outros resultados sobre orientações relacionadas à prevenção de doença e à promoção da saúde, à avaliação concernente à pergunta sobre concordar com o procedimento, às informações sobre possíveis efeitos colaterais do tratamento e à avaliação referente ao fato de o paciente ser questionado se ele tinha alguma pergunta não obtiveram o valor de $p<0,05$ e, por isso, não se apresentaram estatisticamente significativos.

\section{Limitações}

Quando se avaliou a presença de viés de seleção, percebeu-se que só houve diferença significativa na distribuição das variáveis intervenientes entre clínica médica e clínica cirúrgica para gênero $(p=0,04)$.

Positivamente, o questionário passou por uma prétestagem com 15 pacientes, para eliminar possíveis questões confundidoras, apesar de não ter sido feito procedimento de validação (análise do construto e do poder discriminante).

Dois pontos de possível geração de vieses foram a falta de registro dos dados de pacientes que não quiseram participar da pesquisa e o fato de o questionário aplicado ser dicotômico, o que pode ter limitado a definição da amostragem do hospital naquele momento e uma análise mais precisa da opinião dos pacientes, respectivamente. Entretanto, o fato de o questionário ser de sim/não em quase sua totalidade foi importante para facilitar a aplicação, por ser mais prático e menos demorado, o que era um estímulo ao paciente na decisão em participar do estudo. Além disso, evitou dúvidas maiores sobre qual resposta ofertar, por não ter inúmeras opções.

Em relação ao tipo de especialidade, alguns pacientes tiveram dificuldades em definir qual seria a melhor opção, já que receberam tratamento de equipe variada, passando tanto por procedimentos clínicos como por procedimentos cirúrgicos. Nesses casos, foi solicitado que o paciente avaliasse o médico com quem teve contato por maior tempo para avaliação da comunicação médica.

\section{Análise de sensibilidade}

Para anular as diferenças observadas nas questões "O médico disse o nome dele", O paciente foi informado sobre como será seu tratamento,, “O médico explicou sobre a necessidade da internação" e "Que nota você daria para a comunicação geral do médico?", seria necessário que os indivíduos que se recusaram a responder à pesquisa representassem uma quantidade equivalente ao mínimo de $20 \%$ dos pacientes clínicos e $20 \%$ dos pacientes cirúrgicos entrevistados, e tivessem uma opinião ou uma nota oposta à tendência observada na sua categoria (clínico ou cirúrgico), quais sejam: sim no lugar de não e vice-versa, e 4,46 no lugar de 4,0 e vice-versa. Apesar de não ter sido registrada, essa taxa de recusa é pouco provável, já que foi mínima a recusa dos pacientes - cerca de dois ou três na clínica médica e nenhum na clínica cirúrgica decidiram não participar do estudo. A impressão dos entrevistadores é de que a amostra era homogênea entre os participantes e não participantes, já que eram pacientes do mesmo grupo etário da maioria do estudo, utilizando o mesmo sistema de saúde, atendidos por equipe médica igual e realizando procedimentos semelhantes aos demais pacientes.

\section{Generalização}

O CHS é um hospital universitário que dispõe de profissionais médicos altamente qualificados (docentes, preceptores e assistentes), além de residentes e internos, com forte presença nos serviços. Também é hospital-escola para outras profissões da saúde. Entretanto, apesar de ser hospital universitário, é público, o que restringe suas possibilidades quando comparado a hospitais privados de alta complexidade. Isso pode impactar mais fortemente procedimentos altamente dependentes desses recursos, tal como a clínica cirúrgica.

Sendo o campo de coleta de dados um hospital com casos mais complexos e já triados pelo sistema da Central de Regulação de Ofertas de Serviços de Saúde (CROSS) ou do Serviço de Atendimento Móvel de Urgência (SAMU), os dados obtidos podem não ser generalizáveis para todos os níveis de saúde e não representam a população da região, já que o CHS é uma referência regional para determinadas especialidades, não todas.

Avaliando o nível de complexidade dos tratamentos ofertados no CHS, estudos indicam que a maioria dos profissionais da equipe de saúde releva incômodo e despreparo para o momento de conversa com o paciente diante de doenças graves e ameaçadoras da vida ${ }^{27,28}$. Além disso, é preciso considerar a capacidade individual de tolerância de cada indivíduo para a transmissão de más notícias, tanto em relação a diagnóstico, prognóstico e cura, como sobre terapêuticas ${ }^{29}$.

Por isso, para uma generalização adequada dos resultados encontrados, novos estudos precisam ser realizados em níveis diferentes do cuidado médico com o objetivo de avaliar a 
percepção dos pacientes sobre a comunicação médica, os quais podem ter um olhar mais abrangente dos grupos entrevistados, tamanho amostral e eliminação de possíveis vieses.

\section{CONCLUSÃO}

Tendo em vista a relevância do sujeito estudado e os déficits ainda presentes na comunicação médica, é essencial abordar a questão com profissionais da área, especialmente durantea graduação, enfatizando a importância da comunicação para o desenvolvimento de um bom relacionamento entre médico e paciente e seu fator de impacto na qualidade de vida do indivíduo durante a hospitalização. Por isso, na educação médica, é necessário ter investimento em intervenções que visem ao desenvolvimento da habilidade de comunicação.

\section{AGRADECIMENTOS}

Agradecemos ao Conselho Regional de Medicina do Estado de São Paulo (Conselho Regional de Medicina do Estado de São Paulo) e ao Conjunto Hospitalar de Sorocaba (Complexo Hospitalar de Sorocaba).

\section{CONTRIBUIÇÃO DOS AUTORES}

Todos os autores trabalharam igualmente na construção do artigo.

\section{CONFLITO DE INTERESSES}

Os autores declaram não haver conflito de interesses neste estudo.

\section{FINANCIAMENTO}

Este projeto foi apoiado pelo Conselho Regional de Medicina do Estado de São Paulo (Cremesp).

\section{REFERENCES}

1. Brasil. Diretrizes Curriculares Nacionais dos Cursos de Graduação em Enfermagem, Medicina e Nutrição [Internet]. Brasília: Conselho Nacional de Educação; 2001 [access in 22 oct. 2020]. Available from: http://portal. mec.gov.br/dmdocuments/ces1133.pdf.

2. Stewart $M$, Brown J, Weston WW, McWhinney IR, McWilliam CL, Freeman TR. Patient-centered medicine: transforming the clinical method. 2nd ed. Oxon: Radcliffe; 1995.

3. Ong LM, De Haes JC, Hoos AM. Doctor-patient communication: a review of the literature. Soc Sci Med. 1995;40:903-18.

4. Conselho Federal de Medicina. Recomendação $n^{\circ} 1$, de 21 de janeiro de 2016 [access in 21 oct 2020]. Available from: https://portal.cfm.org.br/ images/Recomendacoes/1_2016.pdf.

5. Loge JH, Kaasa S, Hytten K. Disclosing the cancer diagnosis: the patient's experience. Eur J Cancer. 1996;33(6):878-8.

6. Scott JT, Harmsen M, Prictor MJ, EntwistleVA, Sowden AJ, Watt I. Recordings or summaries of consultations for people with cancer. Cochrane Database Syst Rev. 2003;(2):CD001539. doi: 10.1002/14651858.CD001539.

7. Zolnierek KB, Dimatteo MR. Physician communication and patient adherence to treatment: a meta-analysis. Med Care. 2009;47(8):826-34.
8. Freitas $\mathrm{H}$, Oliveira $M$, Saccol A, Moscarola J. O método de pesquisa survey. RAUSP Manage J. 2000;35(3):105-12.

9. Governo do Estado de São Paulo. Conjunto Hospitalar de Sorocaba conta com novo modelo de gestão. 2018 [access in 28 jul 2020]. Available from: http:// aquietrabalho.com/deputados-do-pdo-fiscalizam-hospitais-em-sorocaba/.

10. Brasil. Resolução $n^{\circ} 3$, de 20 de junho de 2014. Institui Diretrizes Curriculares Nacionais do Curso de Graduação em Medicina. Brasília: Ministério da Educação, Conselho Nacional de Educação; 2014 [access in 22 oct 2020]. Available from: http://portal.mec.gov.br/index php?option $=$ com_docman\&view $=$ download\&alias $=15874$-rces003 14\&category_slug=junho-2014-pdf\&ltemid=30192.

11. StataCorp. Stata 16.0. Texas: StataCorp; c1985-2019.

12. Oliveira V, Pimentel D, Vieira M. O uso do Termo de Consentimento Livre e Esclarecido na prática médica. Rev Bioét. 2010;28(3):705-24.

13. Razera A, Braga E. A importância da comunicação durante o período de recuperação pós-operatória. Rev Esc Enferm USP. 2011;45(3):632-7.

14. Singer $\mathrm{S}$, Gotze $\mathrm{H}$, Mobius $\mathrm{C}$, Witzigmann $\mathrm{H}$, Kortmann R-D, Lehmann A, et al. Quality of care and emotional support from the inpatient cancer patient's perspective. Langenbeck's Arch Surg. 2009;394(4):723-31.

15. Schaller M, Murray D. Pathogens, personality, and culture: disease prevalence predicts worldwide variability in sociosexuality, extraversion, and openness to experience. J Pers Soc Psych. 2008;95(1):212-21.

16. Feba $F$, Marques $S$. Perfil de personalidade do médico cirurgião e do médico pronto-socorrista: um estudo exploratório. Psicol Form. 2010;14:44-69.

17. Kang S, Boo Y, Lee J, Han HJ, Jung CW, Kim CS. High occupational stress and low career satisfaction of Korean surgeons. J Korean Med Sci. 2015;30(2):133-9.

18. Bellodi P. Clínica ou cirurgia: um estudo sobre razões da escolha da especialidade. Med J. 2004;122(3):81-6.

19. Bohrer T, Koller M, Schlitt H, Bauer H. Quality of life of German surgeons: results of a survey of 3,652 attendees of the annual meetings of the German Surgical Societies. Dtsch Med Wochenschr. 2011;136(42):2140-4.

20. Dosanjh S, Barnes J, Mohit Bhandari M. Barriers to breaking bad news among medical and surgical residents. Med Educ. 2001;35(3):197-205

21. Roter $D$. The enduring evolving nature of the patient-physician relationship. Patient Educ Couns. 2000;39:5-15.

22. Sheppard V, Williams K, Harrison T, Jennings Y, Lucas W, Stephen J, et al. Development of decision-support intervention for black women with breast cancer. Psychooncology. 2010;19(1):62-70.

23. Endo C, Akechi T, Okuyama T, Seto T, Kato M, Seki N, et al. Patient-perceived barriers to the psychological care of Japanese patients with lung cancer Jpn J Clin Oncol. 2008;38(10):653-60.

24. Arora N, Gustafson D. Perceived helpfulness of physicians' communication behavior and breast cancer patients' level of trust over time. J Gen Intern Med. 2009;24(2):252-5.

25. Ruberton PM, Huynh HP, Miller TA, Kruse E, Chancellor J, Lyubomirsky S. The relationship between physician humility, physician-patient communication, and patient health. Patient Educ Couns. 2016;99(7):1138-45.

26. Conselho Federal de Medicina. Código de Ética Médica. Brasília, DF: CFM; 2010.

27. Ribeiro MCSA, Barata RB, Almeida MF, Silva ZP. Perfil sociodemográfico e padrão de utilização de serviços de saúde para usuários e não-usuários do SUS - PNAD 2003. Cienc Saude Colet. 2006;11(4):1011-22.

28. Mattos JM, Mendonça MHLC. A revelação do diagnóstico de HIV/AIDS à criança e ao adolescente. In: Padoin SMM, organizador. Experiências interdisciplinares em AIDS: interfaces de uma epidemia. Santa Maria: UFSM; 2006. p. 187-204.

29. Nápoles BM, Babié PT, Gutiérrez JA, Jordán MG, Toirac RR. Conocimiento del diagnostico en pacientes con cáncer antes de recibir tratamiento oncoespecífico en 1997. Rev Cuba Oncol. 2000;16(2):93-9.

This is an Open Access article distributed under the terms of the Creative Commons Attribution License, which permits unrestricted use, distribution, and reproduction in any medium, provided the original work is properly cited. 